\title{
Dye Laser
}

National Cancer Institute

\section{Source}

National Cancer Institute. Dye Laser. NCI Thesaurus. Code C64722.

A laser in which light is emitted by a fluorescent organic dye and which can be tuned to radiate at any of a wide range of frequencies. 